\title{
Endoscope-assisted retrosigmoid infralabyrinthine approach to jugular foramen tumors
}

\author{
Madjid Samii, MD, PhD, 1,2 Maysam Alimohamadi, MD,, ${ }^{1,2}$ and Venelin Gerganov, MD, PhD' \\ ${ }^{1}$ International Neuroscience Institute, Hannover, Germany; and ${ }^{2}$ Brain and Spinal Cord Injury Research Center (BASIR), \\ Neuroscience Institute, Tehran University of Medical Sciences, Tehran, Iran
}

\begin{abstract}
OBJECTIVE Removal of jugular foramen (JF) tumors usually requires extensive skull base approaches and is frequently associated with postoperative morbidities such as lower cranial nerve injury. The endoscope-assisted retrosigmoid infralabyrinthine approach is a relatively new approach to tumors extending into the bony canal of the JF. The authors present their experience with this approach.

METHODS The endoscope-assisted retrosigmoid infralabyrinthine approach was used in 7 patients, including 5 with schwannomas and 2 with paragangliomas. The access to the tumor, extent of its removal, postoperative neurological outcome, and approach-related morbidities were evaluated.

RESULTS Two patients had a history of previous partial tumor removal, and 1 was treated by embolization followed by two courses of Gamma Knife radiosurgery. In this latter patient near-total resection was achieved. Gross-total resection was possible in the remaining 6 patients. Five patients benefited from endoscopic assistance: in 2 patients it showed a tumor remnant after microscopic tumor removal, while in 3 patients it allowed safe removal of the intraforaminal tumor by visualizing the surrounding structures. No permanent neurological deficit was observed after the operation. Two patients presenting with swallowing disturbance had temporary postoperative worsening that improved later. One patient developed CSF leakage that was managed with a lumbar drain.
\end{abstract}

CONCLUSIONS This study shows that the judicious application of the endoscope-assisted retrosigmoid infralabyrinthine approach is safe and effective for removal of the schwannomas extending into the JF and selected paragangliomas without significant luminal invasion of the sigmoid-jugular system.

http://thejns.org/doi/abs/10.3171/2015.3.JNS142904

KEY WORDS endoscope-assisted; infralabyrinthine; jugular foramen; retrosigmoid; surgical technique

$\mathrm{T}$ HE jugular foramen (JF) is one of the most complex regions of the skull base, which is well-hidden by osseous and meningeal structures and crowded by many neurovascular elements. Tumors originating from or extending to the JF may extend both intra- and extracranially through the canal of the $\mathrm{JF}^{3,4,6,11,17}$ A variety of surgical approaches to these tumors have been described, depending on the anatomical extension of the tumor, venous anatomy of the JF, and ultimate goal of the surgery. $3,4,11$ Traditional skull base approaches to these areas, such as petrosal approaches, transcondylar approaches (with su- prajugular, retrojugular, and transjugular variants), and lateral skull base (Fisch) approaches endanger hearing and facial nerves. Furthermore, these approaches necessitate extensive bone drilling and sometimes ligation of the sigmoid sinus (SS) and jugular vein. ${ }^{14}$ The senior author's strategy for JF tumors since the mid-1980s has been to remove the cerebellopontine tumor part via a retrosigmoid craniotomy, followed by drilling of the posterosuperior wall of the JF to access the intraforaminal tumor part. ${ }^{13}$ Although part of the tumor might thus be removed, more anteromedially extending tumors remain inaccessible.

ABBREVIATIONS CN = cranial nerve; IJV = internal jugular vein; IPS = inferior petrosal sinus; JB = jugular bulb; JF = jugular foramen; SS = sigmoid sinus. SUBMITTED December 22, 2014. ACCEPTED March 30, 2015. 
In this article, we present a new endoscope-assisted extension of the retrosigmoid approach to the lesions extending into the JF. We present our experience in using this technique and evaluate tumor accessibility, completeness of tumor removal, and outcome. The particular focus of this study was approach-related morbidities and cranial nerve $(\mathrm{CN})$ functional outcome.

\section{Methods}

We retrospectively reviewed all patients with JF tumors treated by the endoscope-assisted retrosigmoid infralabyrinthine approach at our center from 2005 to 2013. All patients underwent operations with the aim of complete tumor removal. The extent of resection was determined according to the surgeon's impression during the surgery, which was further confirmed by postoperative imaging reviewed by an independent neuroradiologist. Complete resection of the tumor was graded as gross-total resection. Near-total resection was defined as the existence of a residual part of the tumor consisting of less than $5 \%$ of the tumor mass around the neurovascular elements, while a residue of 5\% or larger was considered subtotal resection. The main outcome measures of this study were the extent of tumor removal, approach-related morbidities, and neurological outcome.

Postoperatively all patients underwent an early brain CT scan within the first 48 hours after the operation and brain MRI to evaluate the extent of resection. Follow-up was conducted by clinical and imaging examination of the patients, with the first visit occurring within the first 3 months after the surgery.

\section{Surgical Anatomy \\ Osseous Structures}

The JF is located at the caudal end of the petroclival fissure (Fig. 1 left) and is thus sometimes referred to as the posterior foramen lacerum..$^{20}$ The JF is not a true foramen; it is rather a canal with endocranial and exocranial openings and courses inferiorly and anterolateraly under the petrous bone..$^{11,18,20}$ This canal is triangular-shaped and bounded by the petrous part of the temporal bone anterolaterally and the basioccipital bone posteromedially. ${ }^{18-20}$ The features of anterolateral margin of the JF formed by the petrous bone are named (from posterior to anterior) the jugular notch, the intrajugular process, and the pyramidal fossa and sulcus for the inferior petrosal sinus (IPS). The jugular notch is the anterior border of the jugular fossa that contains the jugular bulb. The intrajugular process or spine of the temporal bone is a bony septum running from an anterolateral to posteromedial direction and divides the JF into a smaller anteromedial and a larger posterolateral compartment. The pyramidal fossa, or glossopharyngeal recess, is located at the superomedial end of the JF and is bound by the cochlear aqueduct superolaterally. The part of the basiocciput that forms the medial edge of the JF is the jugular tubercle.

\section{Meningeal Structures}

The dura mater of the endocranial opening of the JF forms a thickened lip that runs from a superolateral to inferomedial direction and is called the plica occipitalis oblique, or jugular dural fold. It covers the intrajugular process, the glossopharyngeal recess, and the $\mathrm{CN}$ meatus. ${ }^{11,17,19}$ The dural covering of the JF has a defect called the jugular pocket, through which the lower CNs exit the cranium. ${ }^{11,17}$ The glossopharyngeal meatus is located anterosuperomedially and is separated by a dural septum (Fig. 1 right) from the vagal meatus that contains the vagus and accessory nerves. ${ }^{1,11,17,19}$ Inside the canal of the JF the lower CNs are tightly enveloped in a common sheath of periosteal dura that gradually transforms into epineurium. ${ }^{17}$

\section{Vascular Structures}

The only arterial branch that exists in the JF is the posterior meningeal artery, which mostly originates from the ascending pharyngeal artery (and in $<10 \%$ from the
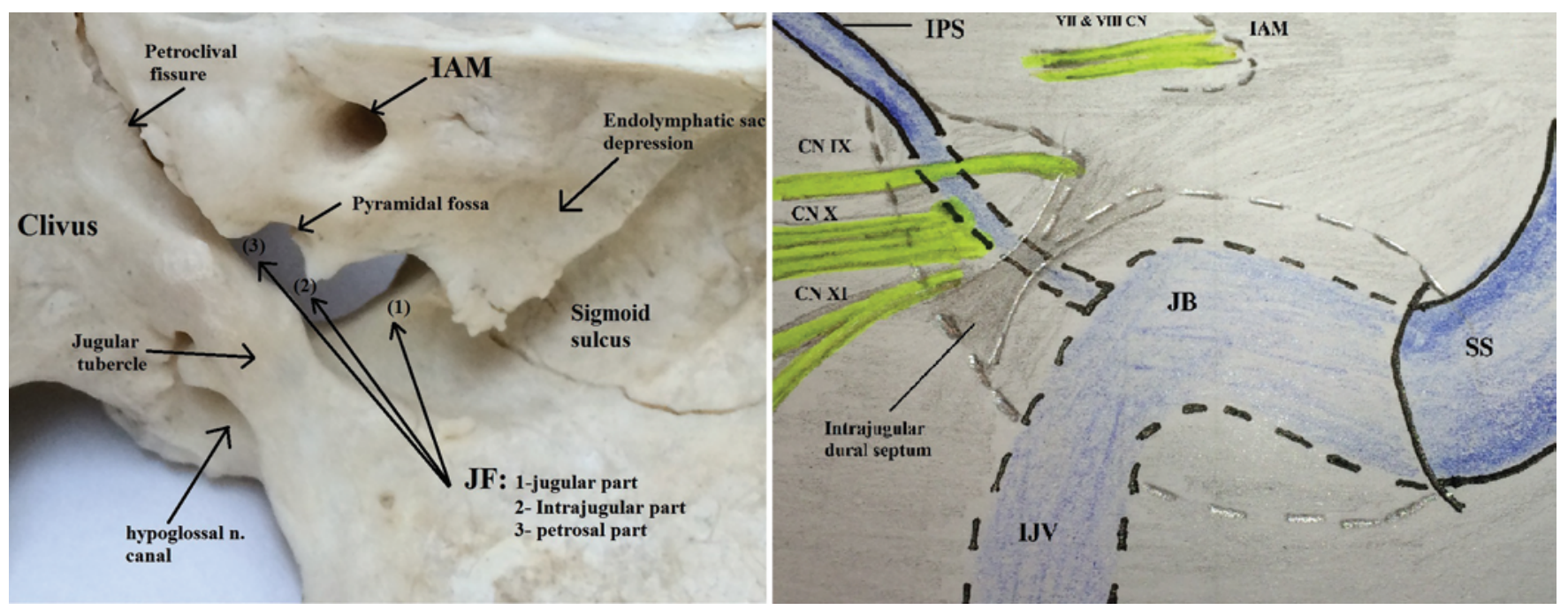

FIG. 1. Left: Bone landmarks in and around the left JF. Right: Schematic drawing of the venous and neural structures at the endocranial opening of the JF. The dashed line demarcates the bone limits of the JF. IAM = internal auditory meatus. Figure is available in color online only. 
anterior inferior cerebellar artery). The posterior meningeal artery is usually located between the X and XI lower CNs. ${ }^{12,18-20}$ The 2 major venous structures inside the JF are the IPS and the jugular bulb (JB; Fig. 1 right). The IPS lies in the petroclival fissure and drains into the JB between the IX and $\mathrm{X}$ lower CNs through multiple venous channels.1,10,19 The JB is located in the jugular fossa and lies posterolateral to the intrajugular process and the lower CNs. The 3 groups of draining veins into the JB are the IPS that drains into the JB from the anterior aspect, the SS that drains into the JB from the posterolateral aspect, and the condylar emissary veins and vertebral venous plexus that drain into the inferior portion of the JB and into the internal jugular vein (IJV).,10,16 Anteriorly, the JB is separated from the internal carotid artery by a thin plate of bone called the caroticojugular spine..$^{10}$

\section{Neural Elements}

Hovelacque classified the JF into the anteromedial compartment, called the pars nervosa, containing CN IX and the IPS, and the posterolateral larger compartment, called the pars venosa, that contains CNs X and XI, the $\mathrm{JB}$, and the posterior meningeal artery. ${ }^{7}$ However, due to the highly variable anatomy of the JF, the most recent anatomical studies do not agree with such a discrete compartmentalization. For surgical purposes we agree with the classification of Katsuta et al. who divided the JF into the petrosal part, the intrajugular part, and the sigmoid part. ${ }^{1,9-11,13,16,18}$ The intrajugular part is located between the two venous parts and contains the meatuses of all the 3 lower CNs in the JF.

\section{Surgical Technique}

For the operation, the patient is fixed in a semisitting (lounging) position, with the head slightly anteflexed and ipsilaterally rotated. The surgery is performed under continuous electrophysiological monitoring of the facial and cochlear nerves, CNs IX-XI (CN X only in selected cases), as well as somatosensory evoked potentials. The skin incision is slightly curved and extends from the top of the external ear down to the neck. A retrosigmoid craniectomy is performed to expose the edges of the transverse sinus and SS and their junction. The dura is opened with a C-shaped incision a few millimeters away from the SS. In contrast to operating on vestibular schwannomas, the dural incision for JF tumors should not necessarily be continued to the level of the transverse sinus. Cerebrospinal fluid is drained from the lateral cerebellomedullary cistern and the cerebellum and dura are retracted medially. The cerebellopontine part of the tumor is debulked with an ultrasonic aspirator and dissected meticulously from the CNs in the arachnoid planes. After complete removal of this part of the tumor, the dura is incised at the posterosuperior aspect of the JF. The dural incision is limited medially by the IPS and petroclival fissure, laterally by the endolymphatic sac depression, and superiorly by the cochlear aqueduct. Using a high-speed diamond drill, the petrous bone is drilled at the jugular notch, intrajugular process, and (if necessary) pyramidal fossa, in a technique similar to drilling of the internal auditory canal for removal of vestibular schwannomas (Fig. 2). Bone drilling is

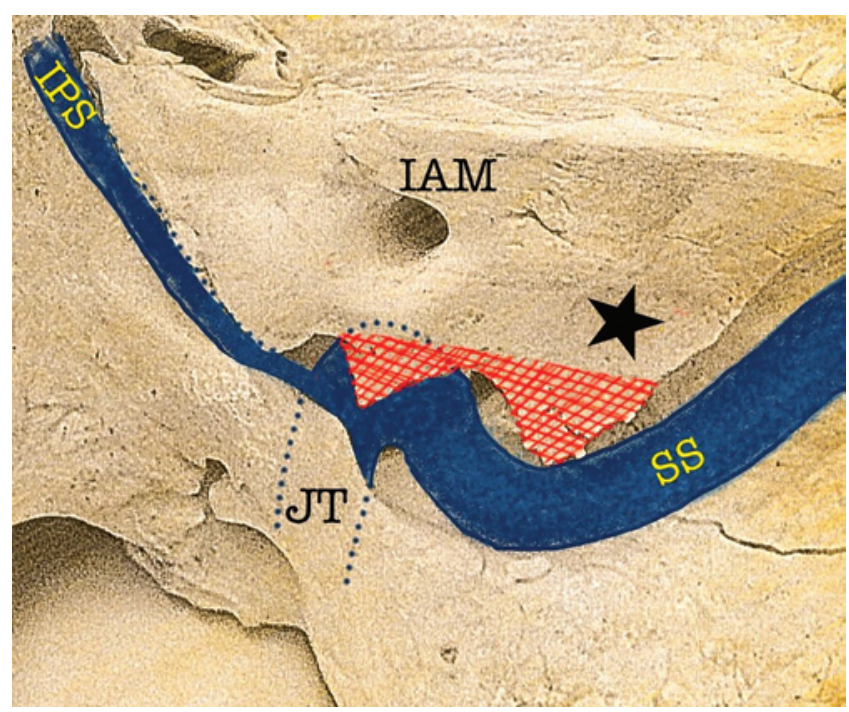

FIG. 2. Photograph of the dry skull showing the bone drilling area (red hatched area) in relation to the osseous and venous landmarks. The star shows the depression of the posterior petrosal surface that underlies the location of the endolymphatic sac. IAM = internal auditory meatus, JT = jugular tubercle. Figure is available in color online only.

tailored to the pattern of tumor extension into the JF and the degree of bone erosion on the thin-slice bone window CT scan in each patient, and could be minimal when the JF is already widened by the tumor itself. This extended exposure of the JF allows removal of the intraforaminal tumor part. During the operation, $0^{\circ}$ and $30^{\circ}$ endoscopes are used to obtain an optimized view while dissecting the intraforaminal tumor from the lower CNs. The endoscopic technique allows visualization and safe removal of the intraforaminal tumor remnants not safely removable under the microscopic view. The endoscope is inserted under microscopic guidance to avoid injury to the surrounding structures. The endoscopes (Karl Storz and Co.) are $4 \mathrm{~mm}$ in diameter, $18 \mathrm{~cm}$ long, and connected to a light source through a fiberoptic cable and to a camera connected to a 21 -inch monitor. The endoscope is held by the primary surgeon and maneuvered in a posterolateral to anteromedial direction toward the JF. In cases in which a tumor remnant is visualized inside the JF by the endoscope, it is dissected and removed using appropriate angled instruments under endoscopic control. During tumor removal from inside the JF, the position of the internal carotid artery can be localized using neuronavigation and a microDoppler probe. After complete tumor removal, the bone defect is covered by fat and sealed by fibrin glue to avoid CSF leakage. Hemostasis is confirmed by jugular compression and the dura is closed in a watertight fashion. The mastoid air cells, if opened, are meticulously packed with fat and fibrin glue. A cranioplasty is completed using methylmethacrylate and the wound is closed.

\section{Results}

\section{Patient Characteristics}

We operated on 7 patients using this technique, including 1 female and 6 male patients, with an average age of 48.4 years old (range 30-65 years old). The tumor type 
was identified as a schwannoma in 5 patients and paraganglioma in 2 patients. The average follow-up period was 25 months (range 13-46 months). The most common presenting symptom was hearing loss (4/7) followed by swallowing disturbance (3/7) and ataxia (2/7). Facial paresis (House-Brackmann Grade IV), facial hypesthesia, and symptoms of raised intracranial pressure were noted in 1 patient each. During clinical examination, sensorineural hearing loss was found in 5 patients and a decreased gag reflex in 3. Two patients (with schwannoma and paraganglioma) underwent operations elsewhere: the tumors had been resected partially and showed regrowth during follow-up examination. One of the patients (with paraganglioma) had also received 2 courses of Gamma Knife radiosurgery.

\section{Exposure and Tumor Removal}

The extent of required bone drilling was determined by the amount of JF erosion by the tumor. In 2 cases with intraforaminal schwannomas it was necessary to drill the whole suprajugular bone, as noted above (standard drilling); only minimal bone drilling was required in the 4 other patients. In 1 patient (with paraganglioma) there was no need to drill the bone due to the extensive bone erosion. The use of the endoscope extended the access to the tumor part growing into the JF under its roof, anteroinferiorly below the petrous bone. The intraforaminal part of the tumor could be followed anteriorly up to the internal carotid artery and inferiorly down to the exocranial opening of the JF. Under endoscopic visualization the tumor could be dissected from the JB and the lower CNs safely in all but 1 patient who was previously treated with Gamma Knife radiosurgery. To avoid injury to these structures, the tumor part that was densely adherent to the lower CNs and JB was left in place in this case. The lower CNs could be dissected from the tumor under microscopic or endoscopic view and were preserved anatomically in all 7 cases. While endoscopic visualization only confirmed complete removal of the intraforaminal part of the tumor in 2 cases, an unnoticed remnant was detected with the endoscope in 2 other cases. In the remaining 3 cases (including both paraganglioma cases), although the tumor remnant inside the JF was visible with the microscope, safe tumor removal could be achieved only with endoscopic visualization. A gross-total tumor removal was achieved in 6 of the 7 patients as confirmed by an independent neuroradiologist.

\section{Neurological Outcome and Complications}

No new permanent neurological deficit occurred in our patients. Two patients with preoperative swallowing dysfunction experienced temporary deterioration of swallowing that was managed successfully by nasogastric tube and non-oral alimentation for 14 days, until the function recovered. None of the patients required a tracheostomy. The patient with preoperative facial paresis recovered during the follow-up period (House-Brackmann Grade II). The cerebellar ataxia and facial hypesthesia improved in all patients.

One patient developed a temporary CSF leak from the surgical site that was managed successfully by placement of a lumbar drain. There were no approach-related complications.

\section{Illustrative Case}

A 61-year-old man was referred after complaining of nausea, vertigo, and imbalance over the previous 6 weeks. A neurological examination revealed no abnormalities except for a left mild sensorineural hearing loss. CT and MRI revealed a mass in the area of the left JF with large intraosseous extension (Fig. 3). The patient underwent an operation using the endoscope-assisted retrosigmoid infralabyrinthine approach. The major steps of surgery are presented in Figs. 4 and 5. The tumor, which was confirmed to be a schwannoma, was able to be completely removed. The postoperative course was uneventful and the patient was discharged after 9 days without any neurological deficit. Postoperative MRI confirmed total extirpation of the tumor while preserving the patency of the JB (Fig. 6). During the 24 months of follow-up, the patient remained stable without recurrence.

\section{Discussion}

Jugular foramen tumors are closely related to the internal carotid artery, SS, JB, IJV, labyrinth and cochlea, fallopian canal, and lower CNs. ${ }^{3}$ Therefore, the surgical approaches to these tumors are among the most complex approaches to the skull base. . $^{3,18,20}$

Many surgical approaches have been used to access and remove JF tumors. Lateral transtemporal approaches are popular among ear, nose, and throat surgeons. The most popular lateral approaches are the transcochlear,
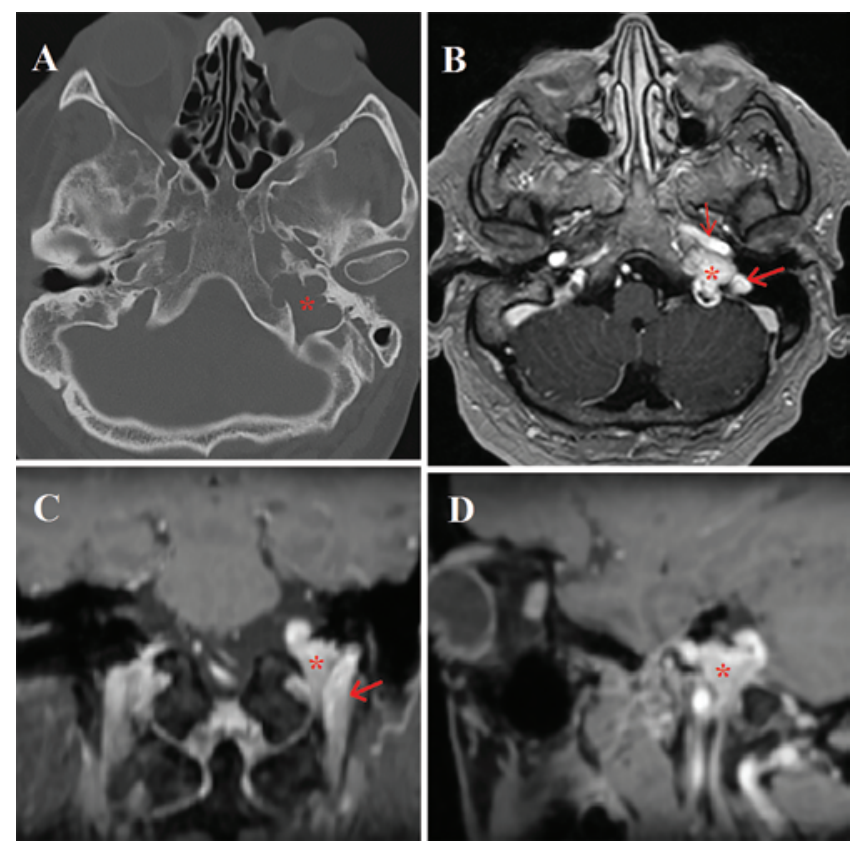

FIG. 3. A: Axial preoperative CT scan of the patient shows widening of the left JF (asterisks) with extension of the erosion to the carotid canal and petrous apex. B-D: Axial, coronal, and sagittal MR images of the patient showing a Gd-enhancing mass in the left jugular fossa, compressing the jugular bulb (thick arrow) and abutting the internal carotid artery (thin arrow). Figure is available in color online only. 

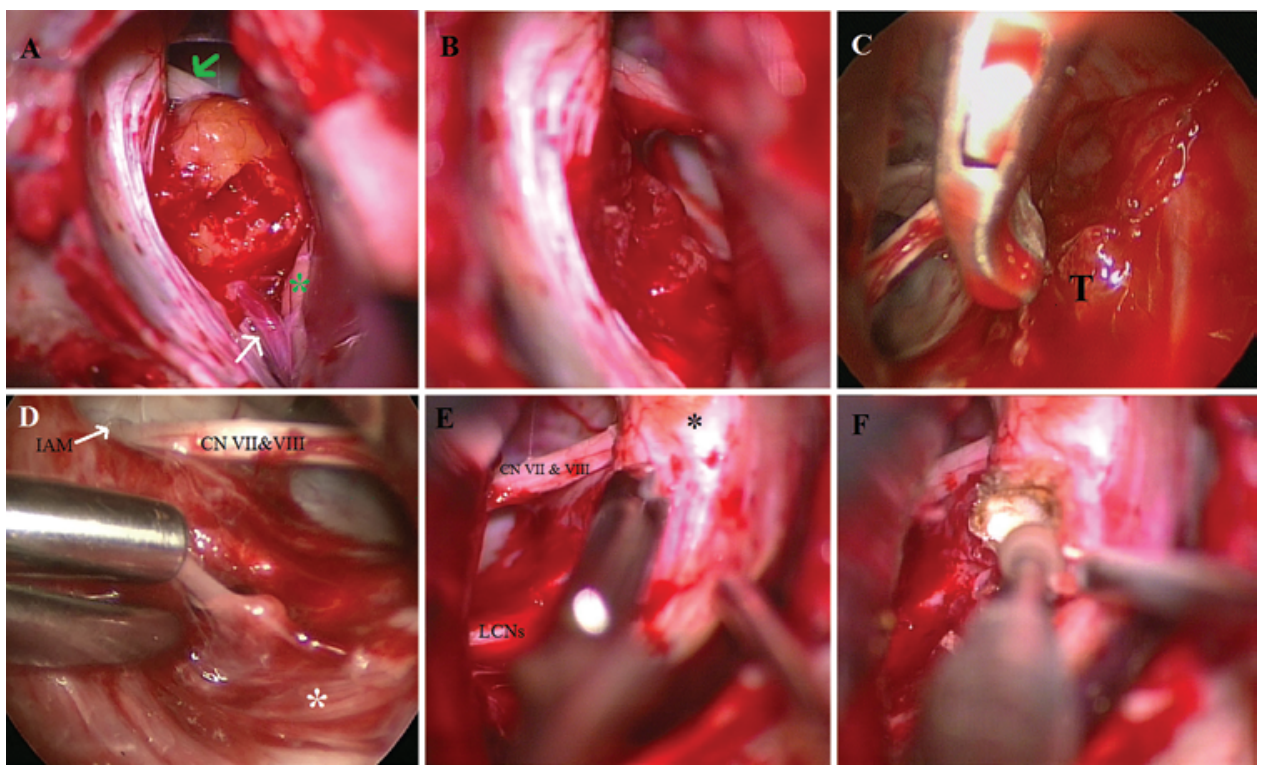

FIG. 4. A: Microscopic view showing the cisternal part of the tumor after partial debulking, compressing the acoustic-facial bundle (green arrow), posterior inferior cerebellar artery (white arrow), and brainstem (asterisk). B: Cisternal part of the tumor is removed under microscopic view. C and D: Under endoscopic view and using angled instruments, the tumor part inside the JF is debulked and the lower CNs (asterisk) are dissected from the tumor (T). E and F: Endoscopic views showing that the dura mater covering the posterosuperior aspect of the JF is incised and the bone drilled away. Asterisk = location of the endolymphatic sac. IAM = internal auditory meatus, LCNs = lower CNs. Figure is available in color online only.

translabyrinthine, and infratemporal approaches. The transcochlear approach sacrifices hearing and requires transposition of facial nerves with the possibility of facial nerve palsy. ${ }^{16}$ The infratemporal fossa approach Type A is probably the most popular approach among ear, nose, and throat surgeons. Despite the outstanding work of Fisch and the others, there are inherent risks of morbidities in the surgical approach, especially facial nerve palsy and conductive hearing loss., ${ }^{2,3,5}$

The transcondylar approach with its variants (suprajugular, retrojugular, and transjugular) has been used by Al-Mefty et al. for JF lesions. ${ }^{8}$ These approaches also need extensive bone drilling and partial removal of the occipital condyle. To expose the tumor extension inside the JF in
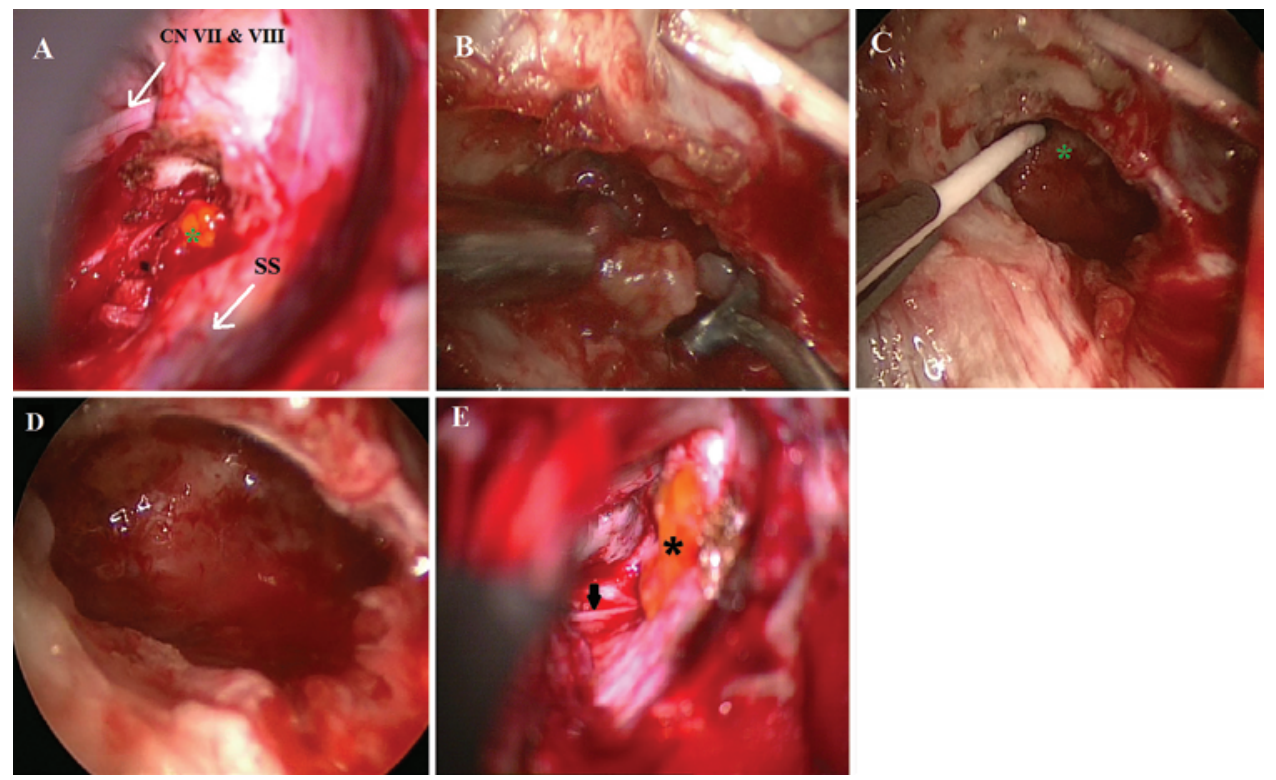

FIG. 5. A and B: After drilling away the suprajugular bone, the tumor (asterisk) inside the JF is exposed and removed using angled instruments. C: The position of the internal carotid artery (asterisk) is checked using a micro-Doppler probe. D and E: The resection cavity is inspected under endoscopic visualization after tumor removal and packed with fat (arrow indicates the preserved lower CNs and the asterisk indicates fat tissue). Figure is available in color online only. 


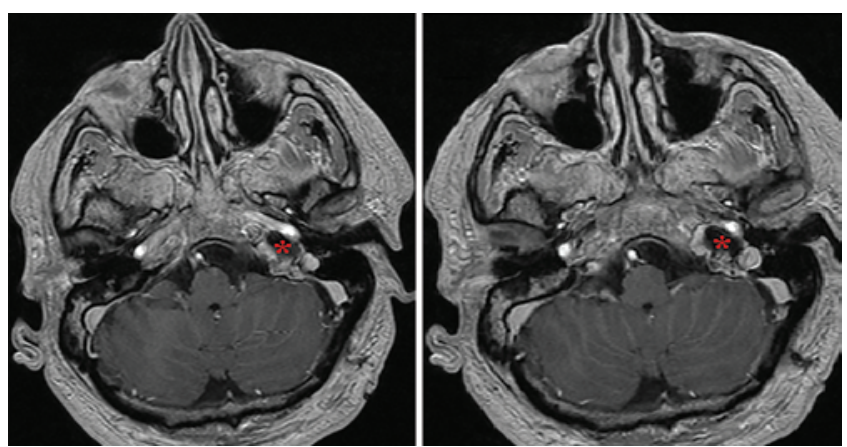

FIG. 6. Early axial postoperative MR images of the patient show tumor removal (asterisk) and restoration of the flow of the JB. Figure is available in color online only.

these approaches, the jugular bulb should be ligated. The authors also state that selection of this approach is dependent on the venous anatomy of the patient, and patency and dominance of the involved JB. ${ }^{6}$

We believe that for benign tumors that do not invade the lumen of the SS and JB, even if preoperative imaging reveals occlusion of the venous outflow at the JF, the patency of these tumors can be restored postoperatively in the majority of cases. Therefore, we do not recommend using venous-sacrificing approaches in these patients. In contrast to schwannomas, paragangliomas tend to invade the lumen of the SS, JB, and IJV more commonly. ${ }^{2,6}$ In these conditions a more extensive approach with exposure of the major vessels in the neck, and sometimes ligation of the SS, JB, and IJV, is necessary for radical removal of the tumor. We recommend application of the "insideout" technique only for selected cases of paragangliomas that do not show significant invasion into the lumen of the sigmoid-jugular system on preoperative imaging.

The presigmoid infralabyrinthine (suprajugular) approach has been discussed by some authors for pathologies of the JF region. ${ }^{2,3,6}$ This approach also requires skeletonizing the SS and extensive drilling of the mastoid process and the fallopian canal to expose the facial nerve, even though facial nerve rerouting is not mandatory. The intradural exposure yielded by this approach is limited, and particularly the cisternal part of the lesion, brainstem, and CNs usually come into view only after a major bulk of the tumor has been removed.

An endoscopic endonasal approach to JF tumors has also been described in some cadaveric studies. ${ }^{4}$ Although this has been theoretically well described, clinical experience with this approach is still very limited and there are only anecdotal reports of its application for patients with JF tumors.

The operative approach to JF tumors should provide adequate access to the area and enable the surgeon to safely remove the tumor. Generous exposure of all the surrounding structures, however, is not a prerequisite for successful surgery. The approach should allow for achieving the goals of surgery but, on the other hand, it should not be a source of approach-related morbidity or major discomfort to the patient. The senior author's strategy for JF tumors since the mid-1980s has been to remove the cerebellopontine tumor part via a retrosigmoid craniotomy, followed by drilling of the posterosuperior wall of the foramen to access the intraforaminal tumor part. ${ }^{14}$ Matsushima et al. described the anatomical steps of this suprajugular approach in detail in a recent cadaveric study. ${ }^{11}$ He has been using the endoscope-assisted retrosigmoid infralabyrinthine approach to JF lesions since 2005 . In this study we described our experience with this approach, in terms of access to the intraforaminal tumor extension, safety of tumor removal, and approach-related morbidity.

Anatomical studies ${ }^{1,17,19}$ have shown that inside the JF the lower CNs are enveloped in a common sheath of the periosteal dura and this makes it difficult to separate them from each other at this segment. Thus, approaching the intraforaminal compartment of the JF tumors from outside (transjugular approach), which necessitates opening of this sheath, endangers the lower CNs. On the other hand, if the sheath is not opened, some tumor residue might be left along the JF that carries a high risk of recurrence. ${ }^{17}$ For instance, in 1 study of the conservative approach, ${ }^{17}$ all of the 6 schwannomas originating from the jugular pocket recurred. Using the endoscope helps to visualize the tumor remnant in the JF from "inside toward outside" and obviates the need to drill the canal of the JF completely and to open the dural sheath of the JF that adds to the risk of lower CN injury. Since the bony wall of the JF is already widened in many cases of JF tumors, only a small area of bone drilling may provide a wider corridor and a wider viewing angle to the most anteromedial part of the JF (Fig. 7). This enlarged corridor makes it possible to apply an endoscope to gain an even closer and magnified view of the JF contents. Comparing to our previous experience with the microsurgical retrosigmoid suprajugular approach, ${ }^{14}$ the endoscope-assisted approach has the advantages of less extensive drilling of the suprajugular bone and a more extended view into the JF. The application of the endoscope during dissection of the tumor from the lower CNs (Fig. 4D) could explain improved outcome in terms of postoperative lower CN function. Furthermore, tumor remnants in the anteromedial part of the JF were detected with the endoscope in $2 / 7$ of our cases. In the other 3 cases safe removal of the intraforaminal part of
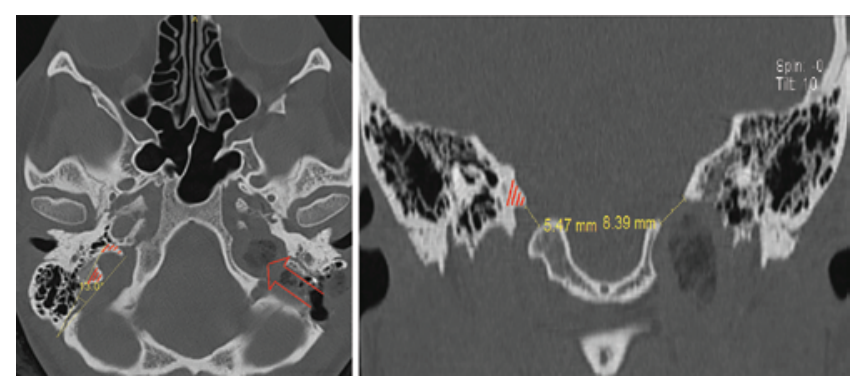

FIG. 7. Axial (left) and coronal (right) CT scans of a patient who underwent an operation through the endoscope-assisted retrosigmoid infralabyrinthine approach on the left side. The axial scan shows widening of the corridor (arrow) to the JF at the operated side. It also depicts how the surgical viewing angle to the anteromedial part of the JF is changed after resection of the hatched area. The coronal section also shows how entry to the JF is made wider after removing the posterolateral wall of the JF. These features make it more feasible to dissect and remove the intraforaminal part of the tumor under endoscopic visualization. Figure is available in color online only. 
the tumor was not possible under the microscopic view and could only be attained under endoscopic visualization. Thus, the endoscopic visualization increased the resection rate in 5 of our 7 cases overall. The limited drilling area allowed avoiding serious CSF leakage, which is particularly troublesome after surgery for JF tumors.

\section{Conclusions}

Our experience with the endoscope-assisted retrosigmoid infralabyrinthine approach shows that its judicious application may be a safe and effective alternative for surgery of tumors extending into the JF, especially when intraluminal growth of the tumor into the sigmoid-jugular system is not present. We suggest application of this approach for JF schwannomas with extension into the canal of the JF, and select cases of JF paragangliomas that do not show significant invasion into the venous system on preoperative imaging.

\section{References}

1. Ayeni SA, Ohata K, Tanaka K, Hakuba A: The microsurgical anatomy of the jugular foramen. J Neurosurg 83:903-909, 1995

2. Borba LA, Ale-Bark S, London C: Surgical treatment of glomus jugulare tumors without rerouting of the facial nerve: an infralabyrinthine approach. Neurosurg Focus 17(2):E8, 2004

3. Cinibulak Z, Krauss JK, Nakamura M: Navigated minimally invasive presigmoidal suprabulbar infralabyrinthine approach to the jugular foramen without rerouting of the facial nerve. Neurosurgery 73 (1 Suppl Operative):ons3-ons15, 2013

4. Dallan I, Bignami M, Battaglia P, Castelnuovo P, Tschabitscher M: Fully endoscopic transnasal approach to the jugular foramen: anatomic study and clinical considerations. Neurosurgery 67 (3 Suppl Operative):ons1-ons8, 2010

5. Fisch U, Fagan P, Valavanis A: The infratemporal fossa approach for the lateral skull base. Otolaryngol Clin North Am 17:513-552, 1984

6. Gjuric M, Bilic M: Transmastoid-infralabyrinthine tailored surgery of jugular paragangliomas. Skull Base 19:75-82, 2009

7. Hovelacque A: Osteologie. Paris: G Doin and Cie, 1967, pp $155-156$

8. Kadri PA, Al-Mefty O: Surgical treatment of dumbbellshaped jugular foramen schwannomas. Neurosurg Focus 17(2):E9, 2004

9. Katsuta T, Rhoton AL Jr, Matsushima T: The jugular foramen: microsurgical anatomy and operative approaches. Neurosurgery 41:149-202, 1997

10. Kveton JF, Cooper MH: Microsurgical anatomy of the jugular foramen region. Am J Otol 9:109-112, 1988
11. Matsushima K, Kohno M, Komune N, Miki K, Matsushima T, Rhoton AL Jr: Suprajugular extension of the retrosigmoid approach: microsurgical anatomy. J Neurosurg 121:397407,2014

12. Rhoton AL Jr, Buza R: Microsurgical anatomy of the jugular foramen. J Neurosurg 42:541-550, 1975

13. Samii M, Babu RP, Tatagiba M, Sepehrnia A: Surgical treatment of jugular foramen schwannomas. J Neurosurg 82:924-932, 1995

14. Samii M, Metwali H, Samii A, Gerganov V: Retrosigmoid intradural inframeatal approach: indications and technique. Neurosurgery 73 (1 Suppl Operative):ons53-ons60, 2013

15. Sanna M, Mazzoni A, Saleh EA, Taibah AK, Russo A: Lateral approaches to the median skull base through the petrous bone: the system of the modified transcochlear approach. J Laryngol Otol 108:1036-1044, 1994

16. Schwaber MK, Netterville JL, Maciunas R: Microsurgical anatomy of the lower skullbase-a morphometric analysis. Am J Otol 11:401-405, 1990

17. Sutiono AB, Kawase T, Tabuse M, Kitamura Y, Arifin MZ, Horiguchi T, et al: Importance of preserved periosteum around jugular foramen neurinomas for functional outcome of lower cranial nerves: anatomic and clinical studies. Neurosurgery 69 (2 Suppl Operative):ons230-ons240, 2011

18. Tekdemir I, Tuccar E, Aslan A, Elhan A, Deda H, Ciftci E, et al: The jugular foramen: a comparative radioanatomic study. Surg Neurol 50:557-562, 1998

19. Tekdemir I, Tuccar E, Aslan A, Elhan A, Ersoy M, Deda H: Comprehensive microsurgical anatomy of the jugular foramen and review of terminology. J Clin Neurosci 8:351-356, 2001

20. Vogl TJ, Bisdas S: Differential diagnosis of jugular foramen lesions. Skull Base 19:3-16, 2009

\section{Disclosure}

The authors report no conflict of interest concerning the materials or methods used in this study or the findings specified in this paper.

\section{Author Contributions}

Conception and design: all authors. Acquisition of data: Alimohamadi. Analysis and interpretation of data: Alimohamadi. Drafting the article: Alimohamadi. Critically revising the article: all authors. Reviewed submitted version of manuscript: all authors. Approved the final version of the manuscript on behalf of all authors: Alimohamadi. Study supervision: Samii, Gerganov.

\section{Correspondence}

Maysam Alimohamadi, Brain and Spinal Cord Injury Research Center (BASIR), Neuroscience Institute, Reyhaneh Building, Imam Khomeini Hospital, Tehran, Iran. email: alimohamadi59@ gmail.com. 